\title{
ADVANCES OF FLASH LIDAR DEVELOPMENT ONBOARD UAV
}

\author{
Guoqing Zhou, Jiazhi Yang, Xin Li and Xiaoping Yang \\ Guangxi Key Laboratory for Spatial Information and Geomatics, Guilin University of Technology, Guilin, 541004, \\ China-glitezhou@yahoo.com
}

Commission VI, WG VI/2 - Automatic Geospatial Data Acquisition and Image-Based Databases

KEY WORDS: UAV, LiDAR, flash, data acquisition, 3D imagery

\begin{abstract}
:
A small cost-low civilian UAV (Unmanned Aerial Vehicle - UAV) platform usually requests that all carried components should be light in weight, small in volume, and efficient in energy. This paper presents the advance of a pre-mature of flash LiDAR system including laser emitting system, associate with the pulsed voltage technology. A complete laser emitting system, including laser diode, conic lens, alignment, divergence angle, etc., has been designed and implemented. The laser emitting system is first simulated and tested using 3D-Tool software, and then manufactured by an industrial company. In addition, a novel power supply topology based on two coupled coils, pulse generator circuit, and a fast switch, is proposed since several $100 \mathrm{~V}$ in voltage, 10-100 A in current, several hundred millisecond in pulse width is needed for flash LiDAR system onboard a small low-cost civilian UAV platform, and the traditional power supply had problems in efficiency and bulk. Finally, laser emitting and the power supply are assembled and tested. The size of laser footprint is $4398.031 \mathrm{~mm}$ x $4398.031 \mathrm{~mm}$ in x and y axes, respectively, when shitting from a flight height of $300 \mathrm{~m}$, which is close to the theoretic size of $4.5 \mathrm{~m} \mathrm{x} 4.5 \mathrm{~m}$. The difference of $102 \mathrm{~mm}$ can meet the requirement of flash LiDAR data collection at a flight height of $300 \mathrm{~m}$. Future work on extensive and on-going investigation and investments for a prototype of flash LiDAR system is drawn up as well.
\end{abstract}

\section{INTRODUCTION}

The 3D imagery has widely been applied in such as terrain mapping, disaster rescue, helicopter obstacle avoidance, range navigation, urban planning, environmental monitoring, resource exploration, and so on. Many technologies have been developed for automatic acquisition of $3 \mathrm{D}$ imagery, such as typical stereo aerial imager onboard airborne. Compared with conventional intensity image captured by optical sensors onboard either airborne or spaceborne, three dimensional (3D) image captured by active sensor offers more information about the target, such as elevation, distance, position and structure (Zhou et al., 2011). Traditionally typical airborne scanning LiDAR (Light detection and Ranging) sensor onboard manned airplane is an active sensor, and is capably of acquiring 3D imagery of an arbitrary object or scene by measuring the time delay of a round trip of a laser pulse directed at points.

In recent years, a UAV platform becomes more and more interesting, since it is capable of quickly reaching the target area and deploying mission to the remote sites at a low-cost. A small low-cost civilian UAV platform often has limitation to its carried components in volume and weight. These limitations have seriously hindered its applicability in practice, since it requires lightness in weight, small in volume, and efficiency in power supply. For this reason, this paper presents an innovative idea about airborne flash LiDAR system. The flash LiDAR systems are analogous to a camera with a flashbulb (flood illumination), but with the flash being provided by laser illumination and the use of a detector with a clock to determine the time it takes for the flash to depart, reflect off of the target, and return $(* * *)$. By measuring the time of flight of the reflected laser pulse, the sensor can determine a range measurement along with intensity for each pixel in the image. This information set over the range of all detector pixels is referred to as a 3D LiDAR imagery (Zhou et al., 2011; Yang and Zhou, 2011).

\section{DESIGNED FLASH LIDAR FLOWCHART}

\subsection{General Framework}

The proposed flash LiDAR onboard a UAV system is depicted in Figure 1. As seen from Figure 1, it consists of five basic functionality modules. They are (1) laser and laser emitting sub-system, (2) APD and laser receiver sub-system, (3) micro-control and processing sub-system, (4) POS subsystem, and (5) LiDAR point cloud postprocessing sub-system. The details of each sub-system are presented by Zhou and Yan (2011). A brief review is presented below.

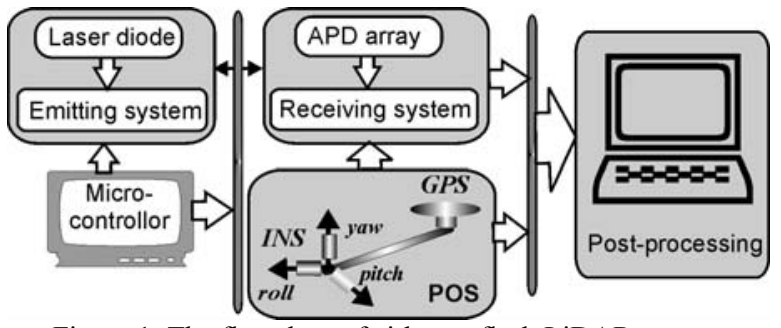

Figure 1. The flowchart of airborne flash LiDAR system 
(1) Laser emitting sub-system: TPGAS2S09H diode laser with wavelength of $905 \mathrm{~nm}$ is used for this project. This laser emitting sub-system is to generate high energy of laser. As a initial research, a prototype with $5 \times 5$ pixels $23 \mathrm{D}$ image array with $<5 \mathrm{~cm}$ ranging accuracy, in real time at rates up to $30 \mathrm{~Hz}$, is designated.

(2) Receiver sub-system: The most common detector used to detect the laser echo pulse is linear mode avalanche photodiode (APD). This research adopted the Geiger-mode APD for receiving photon single. 3D imaging laser radar that uses Geiger-mode APDs is being developed (Aull and Marino, 2005; Daniel, 2003; Johnson, 2003; Aull et al., 2002; Marion et al., 2003). Because of the effect of background light noise, a new system design and new data processing method need to be developed. The details of this sub-system will be reported in the near future.

(3) Micro-Control Sub-system: which is core of system. The microprocessor controls the entire system and enables real-time control laser emitting, receiving, measure of time interval, data sampling, storage, etc. The control device also provides interactive communication with each modules. The control device is, therefore, usually designed as keyboard display unit.

(4) POS subsystem: which is used to provide the attitude of each laser ray and central position of laser emitter for calculation of 3D geodetic coordinates in a given coordination system.

(5) LiDAR point cloud pre-processing: which is used to generate $\mathrm{XYZ}$ coordinates on the basis of range, attitude angles of each laser ray and central coordinates of laser emitter.

\subsection{Principle of Flash Laser Emitting}

A detail of principle for flash laser is given by $\mathrm{Hu}$ (2005). A brief description is given in this Section.

If the size of the emitting plan of a diode laser is $L_{v}$ and $L_{h}$ in length and width, and the divergence angle of the bundle of lasers is $\theta_{v}$, and $\theta_{h}$ in horizontal and vertical plan, the focal length is $f_{t}$, the laser source is located at the focus (see Figure 2).

With geometry in Figure 2, we have

$$
\theta_{t v}=2 \arctan \left(\frac{L_{v}}{2 f_{t}}\right)
$$

Generally, $L_{v}<<2 f_{t}$, so the divergence angle of plan is

$$
\theta_{t v} \approx \frac{L_{v}}{f_{t}}
$$

As observed in Eq. 2, the divergence angle is negatively proportional to the focal length.
To make the direction of emitting laser is parallel to the direction of entry laser, the diameter of aperture must be at least

$$
D_{v l}=L_{v}+2 f_{t} \tan \left(\frac{\theta_{v}}{2}\right)
$$

Usually, $L_{v}<<D_{v l}$, the least diameter of aperture is expressed by

$$
D_{v l} \approx 2 f_{t} \tan \left(\frac{\theta_{v}}{2}\right)
$$

So, the least width of laser plan is

$$
D_{v o}=D_{v l}-L_{v}
$$

Substitute Eq. 3 into Eq. 5, we have

$$
D_{v o}=2 f_{t} \tan \left(\frac{\theta_{v}}{2}\right)
$$

As observed from Eq. 6, the width of laser plan is positively proportional to the focus length.

Combined Eq. 2 and Eq. 6, it is hard to simultaneously meet both conditions, which results in difficulty of designing a reasonable and powerful laser emitter subsystem.

With the same method, we have divergence angle is

$$
\theta_{t h} \approx \frac{L_{h}}{f_{t}}
$$

To make laser power in the emitting system in vertical plan, the least diameter of aperture is

$$
D_{h l} \approx 2 f_{t} \tan \left(\frac{\theta_{h}}{2}\right) \quad(8)
$$

The least width of emitter laser in vertical plan is

$$
D_{h o}=2 f_{t} \tan \left(\frac{\theta_{h}}{2}\right)
$$

For example, if the emitter plan is $225 \times 400 \mu \mathrm{m}$, the divergence angle is $10^{\circ}$ in planar direction and $25^{\circ}$ in vertical direction, and divergence angle is $30-33 \mathrm{mrad}$ after alignment.

With the two cases above, this paper select the fast -axis conical lens with a focal length of $7.7 \mathrm{~mm}$, and $7 \mathrm{~mm}$ and $9 \mathrm{~mm}$ in height and length; select a slow-axis conic lens with a focal length of $13.7 \mathrm{~mm}$, and $13 \mathrm{~mm}$ and $15 \mathrm{~mm}$ in height and length.

The major purpose of the emitting system is to align the emitting laser. Thus, when selecting an emitting laser system, we have to simultaneously consider both the alignment characteristic and the complexity of optical system in structure, processing and manufacturing. With the computational parameters, the $3 \mathrm{D}$ model is simulated using ZEMAX software, as depicted in Figure 3. The simulated 3D model, associated with its size is depicted in Figure 4. 


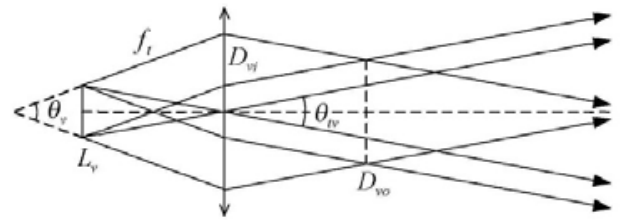

Figure 2. The illustration of divergence angle of laser emitting (Hu, 2005)

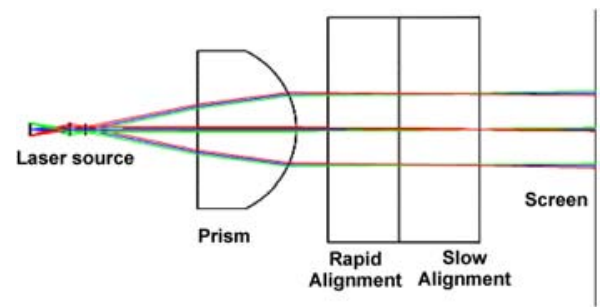

Figure 3. Simulated alignment of laser emitting system

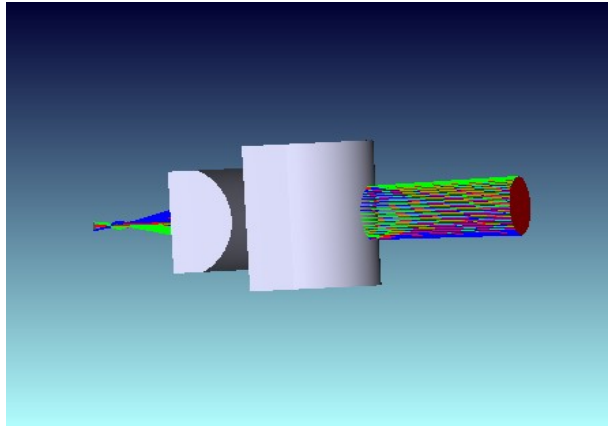

Figure 4. Simulated 3D model for alignment of laser emitting system

\section{COMPONENTS OF LIGHT-EMITING SYSTEM}

\subsection{Laser Diode}

TPGAS2S09H diode laser with wavelength of $905 \mathrm{~nm}$ is used for this project. Diode lasers in the $1300 \mathrm{~nm}$ to 1600 $\mathrm{nm}$ regime are used in a variety of applications including pumping, range finding, materials processing, and aesthetic medical treatments. In addition to the compact size, efficiency, and low cost advantages of traditional diode lasers, high power semiconductor lasers in the eyesafe regime are becoming widely used in an effort to minimize the unintended impact of potentially hazardous scattered optical radiation from the laser source, the optical delivery system, or the target itself.

The laser diode has the following characteristics (see Figure 5):

- Peak power $>300 \mathrm{~W}$ at 30 A driving current and 100 ns pulse width.

- $905 \mathrm{~nm}$ pulsed laser output.

- Extremely high reliability.

- Small emitting areas allow ease of lens or fiber coupling.
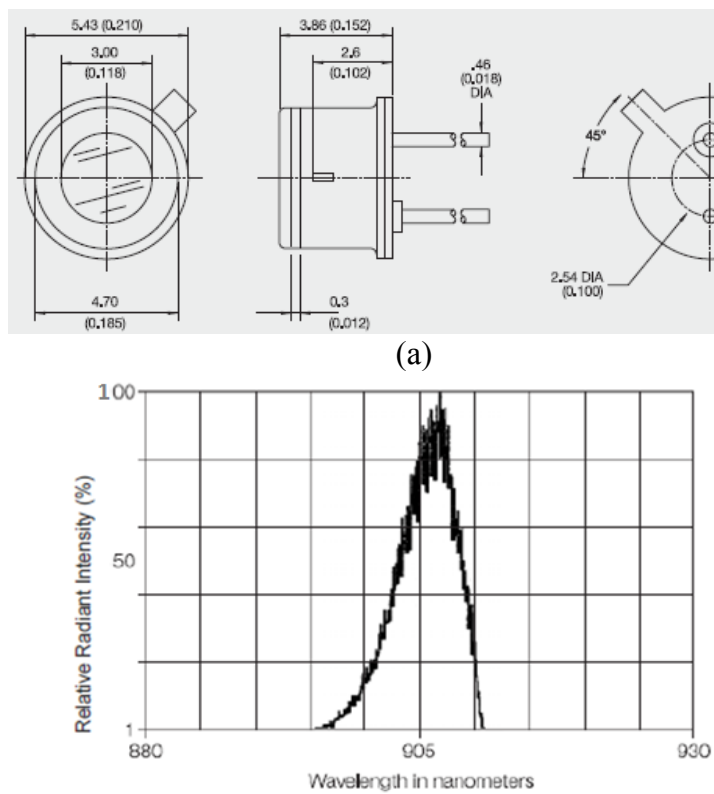

(b)
Figure 5. (a) Package drawing and (b) its spectral plot distribution ( http://www.perkinelmer.com/ CMSResources /Images/44-6562DTS_HighPowerLaser DiodesforRange Finding.pdf)

The junction plane, light-emitting surface and divergence angle of diode laser are depicted in Figure. As seen, the light-emitting surface is not symmetric with the divergence angle. For each light-emitting element, the length in the directtion parallel to junction plane is about $230 \mu \mathrm{m}$, and the height in the direction of vertical to junction plane is $1 \mu \mathrm{m}$. The divergence angles in direction parallel to and vertical to junction plane is $10^{\circ}$ and $25^{\circ}$, respectively. The major functionality of light-emitting optical system of diode laser is aligning and shaping to the bundle of light, which are no-symmetric, big divergence angle and poor quality, to make them meet the requirement of laser, i.e., small divergence angle, alignment and high energetic light.

The final required bundle of laser is: Divergence angles in horizontal and vertical direction is approximately 30 33mrad, the energy Transmittance laser reach above $95 \%$.

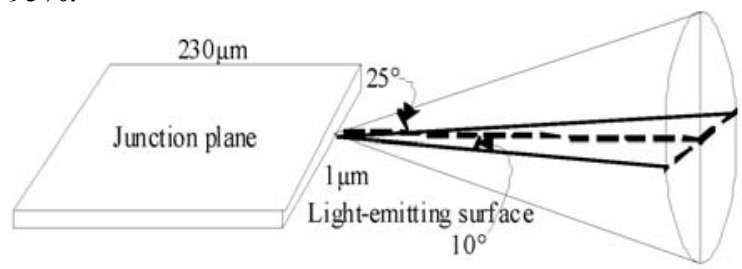

Figure 6. Illustration of ligh-emitting surface and divergencey angle.

This laser emitting sub-system is to generate high energy of laser. As a initial research, a prototype with $5 \times 5$ 
pixels square 3D image array with $<5 \mathrm{~cm}$ ranging accuracy, in real time at rates up to $30 \mathrm{~Hz}$, is designated.

\subsection{Power Driving Circuit for Laser Diode}

A laser diode accompanying with its power supply is an important part of LiDAR system onboard UAV. The traditional power supply had problems in efficiency and bulk, has been demonstrated that it is not proper for application on a small low-cost civilian UAV platform. How to design a power supply for laser diode to meet the requirement of light, small, and energy efficiency, is a valuable work. In this paper, a novel power supply topology for LiDAR system on board UAV platform is presented. The power supply is composed of two coupled coils, pulse generator circuit, and a fast switch (Zhou and Yang, 2011).

Coffey (2009) though that the power-supply largely impacts the performance of laser-diode for a given specification. Different methods of design and implementation of the laser diode power supply have been proposed by Cui et al. (2011), Zhou et al. (2011), Yang et al. (2011). A novel low power supply for DCcoupled $1.25 \mathrm{~Gb} / \mathrm{s}$ laser diode driver is suggested by $\mathrm{Fu}$ et al. (2006). With the MAX797, driver circuit of the high-power laser diode was proposed by $\mathrm{Li}$ and $\mathrm{Xu}$ (2008). For a pulsed power modulated for high output power for laser fuze was proposed by Guo et al. (2011). The automatic power control of DC-coupled burst-mode laser diode was presented by Zhang et al. (2009) and Li et al. (2008).

A proposed schematic diagram of the power supply for laser diode is depicted in Figure 7. As seen from Figure 7, Driving power supply is composed of two coupled coils (could be replaced by a pulsed transformer), a thyristor, TTL pulse signal, resister and capacitor [3-6]. With this topology of power supply, the input voltage of the power supply is a $+28 \mathrm{~V}$ DC voltage from airplane, and the output maximum voltage is $300 \mathrm{~V}$. Before the TTL pulse signal coming, the power supply is in a steady state. During this steady state, capacitor $\mathrm{C} 1$ is charged by the input voltage through $\mathrm{R} 1$ and $\mathrm{L} 1$ to $+28 \mathrm{~V}$; the thyristor Q1 is turn off because the TTL pulse signal is in a low state; there is no current in L2; and the output voltage is 0 . If a pulse signal comes to the gate of the thyristor Q1, Q1 will turn on, and C1 will release its energy through Q1 and L1 rapidly cause the low resistance in the circuit. This situation will generate a high voltage across L2; for L1 and L2 are strongly coupled. The voltage across L2 can be controlled through turns ratio of L2 and L1, here we should choose a turns ratio of 10 . Assuming the other parameters of the two inductors are all the same besides the turns ratio, the inductance of L2 will be 100 times larger than L1. The generated pulsed voltage is around $300 \mathrm{~V}$ (voltage of L1 plus L2). The voltage generated by L2 is coupled to laser diode D1 through R2 and C2.

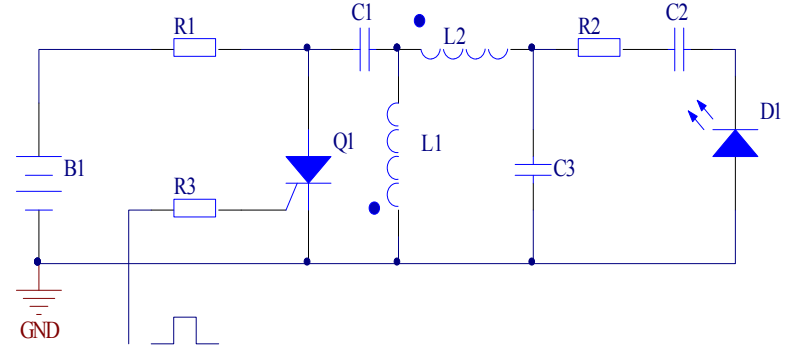

Figure 7. Schematic diagram of the power supply for laser diode

In order to meet the requirement of laser diode adopted in LiDAR scanner onboard small UAV platform, the parameters of each circuit elements have been specified and are depicted in Figure 8. Instead of traditionally trying every element parameter one by one, a circuit model by PSpice is set up and simulation experiment is conducted. This method is fast and cost efficiency.

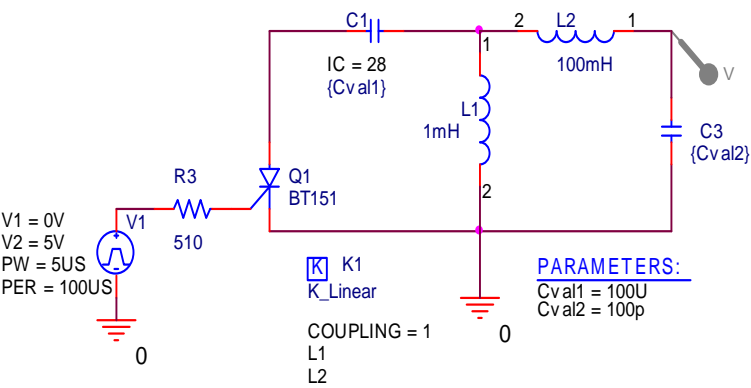

Figure 8. Simulation model of the power supply for laser diode

With the above design, experiments and test, a prototype of power supply is produced, as shown in Fig. 9. The prototype of power supply is synchronous with a pulse signal generated by control circuit, and the output voltage and current adjustable to fit laser diode, and the repeat pulse generation is up to 1000 pulses per second.

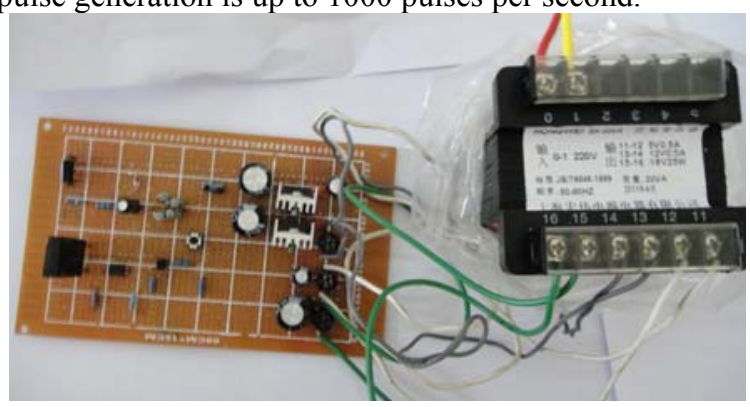

Figure 9. A prototype of power supply

\section{SIMULATED EXPERIMENTS AND RESULTS}

Experiments with simulating different flight height is conducted. One of experimental results with a flight height of $200 \mathrm{~m}$ is shown in Figure 10. As seen from Figure 10, the shape of footprint is a square with a size of at $4398.031 \mathrm{~mm} \times 4398.031 \mathrm{~mm}$ in $\mathrm{x}$ and $\mathrm{y}$ axes, respectively, which is close to the theoretic size of $4.5 \mathrm{~m}$. The difference between practice and theoretic values is $102 \mathrm{~mm}$. However, it is estimated that practical error may 
be bigger than $102 \mathrm{~mm}$ due to error, alignment, atmospheric impact, etc. In addition, the divergence angles in horizontal and vertical directions are almost the same.

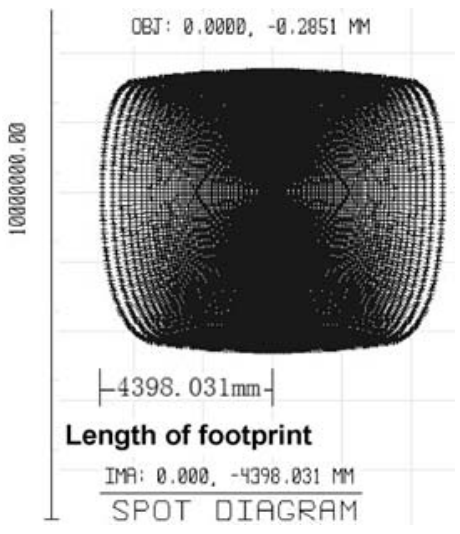

Figure 10. Simulated footprint at a flying height of $300 \mathrm{~m}$ above local average elevation

\section{MANUFACTURES}

The laser emitting device is manufactured by Zhenhong LLC at Dongkuan, Guangdong, China. With the designed parameters by our theoretic analysis, 3D-Tool software is used for the visualization of laser emitting device, as shown in Figure 11. 3D-Tool is a powerful, cost effective tool that has helped customers substantially in the management of manufacturing technologically advanced products. 3D-Tool allows to see, evaluate, measure, cross section.

The emitting laser component consists of basis, front and rear cushion, ring, tube, polysulfone sets, septa, diode laser, as depicted in Figure 11. Terms are as follows.

a1: base for rear lens

a2: base for front lens

a3: isolation piece for separating front and real lens

a4: flatting pieces for stabling the front lens

a5: screw tube for installing and stabling polysulfone

a6: polysulfone for stabling laser diode

b1: cushion (base)

c1: lock ring for stabling tube

d1: laser diode

e1: emitting tube with two lens, front lens and rear lens inside

\section{CONCLUSION}

This paper presents the advances of the flash LiDAR initative with focus on light-emitting system. The proposed flash LiDAR is imaged with the 3D imaging mode, and the entire scene within the sensor's field of view (FOV) at a single flash of the laser. This is because the proposed flash LIDAR have many advantages such as inherently insensitive to ambient and stray light, and the glint and clutter outside of the expected range to the target filtered and discarded. Since the flash LiDAR captures a complete image with each flash. This capability makes data pre-processing and post-processing extremely efficient and reliable since range, bearing, and pose algorithms do not have to deal with interpreting data from glint and clutter. Rapid frame rates, waveband filtering, and time gating on the return signal increase glint tolerance even further. Thus these advantages will bring significant improvement for its application in the traditional fields such as topographic mapping and disaster monitoring.

This paper only reports a pre-mature of flash LiDAR technology. Extensive and on-going investigation and investments for a prototypes of flash LiDAR system is under development. The system will be tested in the laboratory and in-flight airplane and helicopter in field tests.

\section{ACKNOWLEDGEMENTS}

This paper is financially supported by GuangXi Governor Grant under approval number of 2010-169, China Natural Science Foundation under contract number 41162011, GuangXi Grand Natural Science Foundation under contract number, 2011GXNSFD018001, GuangXi Grand Natural Science Foundation under the number of 2012GXNSFCB053005, and the grant of the GuangXi Key Laboratory of Spatial Information and Geomatics under contract number, GuiKeNeng110-31-08-01. The authors would thank those who gave us their hands in experimental design and technical advice.

\section{REFERENCES}

Albota, M. A., Heinrichs, R. M., Kocher, D. G., 2002. "Threedimensional imaging laser radar with a photon-counting avalanche photodiode array and microchip laser," Appl. Opt. 41, pp. 7671-7678.

Aull, B. F., Marino, R. M., 2005. "Three-dimensional imaging with arrays of Geiger-mode avalanche photodiodes," Proc. SPIE 6014, pp. 1-12.

Aull, B. F., Loomis, A. H., Young, D. J., et al, "Geiger-mode avalanche photodiodes for three-dimensional imaging," Lincoln Lab. J. 13, pp. 335-3509.

Baker, R. J. and S. T. Ward, 1994. "Designing nanosecond high voltage pulse generators using power MOSFETs". Electronics Letters. 30 (20): pp.1634-1635.

Coffey, V.C., 2009. Laser-diode power-supply basics: performance depends on specification, Laser Focus World, v 45 , n 2, pp. $60-2$.

Cui, Wei; Chen, Dianren; Yue, Yubo; Chen, Lei; Li, Xingguang, 2011. A novel deign method of laser diode power supply, Applied Mechanics and Materials, v 44-47, pp. 18891892.

Daniel, G. F., 2003. "Detection and false alarm probabilities for laser radars that use Geiger-mode detector," Journal of Geodynamics," Appl. Opt. 42, pp. 5388-5398. 
Degnan, J., McGarry, J., Zagwodzki, T., 2002. " Design and performance of a 3-D imaging, photon-counting, microlaser altimeter operating from aircraft cruise altitudes under day or night conditions," Proc. SPIE, vol. 4546, pp.1-10.

Fu, Sheng-Meng; Chao-Yang Chen, 2006. DC-coupled burstmode laser diode driver with automatic power control for $1.25 \mathrm{Gbit} / \mathrm{s}$ PON system, Analog Integrated Circuits and Signal Processing, v 46, n 2, pp. 159-64.

Guo, Jing; Zhang He; Zhang Xiangjin; Wang Xiaofeng, 2011. Pulsed power modulated driving technology with high output power for laser fuze, 2011 Symposium on Photonics and Optoelectronics, pp. 4.

$\mathrm{Hu}$, Chunshen, 2005. Study on Quick 3D imagery of Laser Diode, Ph.D. dissertation, National Defense University, October, 2005.

Johnson, S., Gatt, P., Nichols, T., 2003. "Analysis of GeigerMode APD Laser Radar," Proc. SPIE, vol. 5086, pp.359368.

Kilpelae ,Ari. and Sergei Vainshtein. 1997. "Generating optical pulses for a fast laser radar". The International Society for Optical Engineering, pp. 237-247.

Li, Manli; Huiying Xu, 2008. Analysis and design of the highpower laser diode drive circuit based on MAX797, Proceedings of the SPIE - The International Society for Optical Engineering, v 7156, pp. $71562 \mathrm{U}$ (8 pp.).

Li, Day-Uei; Huang, Li-Ren; Tsai, Chia-Ming, 2005. A 3.5$\mathrm{Gb} / \mathrm{s}$ CMOS burst-mode laser driver with automatic power control using single power supply, Proceedings - IEEE International Symposium on Circuits and Systems, pp. 55015504.
Marino, R. M., Stephens, T., Hatch, R. E., 2003. "A compact 3D imaging laser radar system using Geiger-mode APD arrays: system and measurements," Proc. SPIE, vol. 5086, pp. 1-15.

Niclass, C., Charbon, E., 2004. "A CMOS Single Photon Avalanche Diode Array for 3D imaging," ISSCC Digest Tech. Papers, pp. 120-121.

Thompson, Marc T. Schlecht, Martin F. 1997. "High power laser driver based on power converter technology". Power Electronics, (1): pp. 3-4 .

Tullio, Mariani, Carlo Frediani, Cesare Ascoli. 1997. "Simple, Linear, and Safe Light Control for Laser Diodes". Rev. Sci. Instrum., 68 (6): pp. 2594-2595.

Yang, Jiazhi and Guoqing Zhou, 2011. Design and implementation of power supply of high-power diode laser of LiDAR onboard UAV, 2011 IEEE International Symposium on Image and Data Fusion, Tengchong, Yunnan Province, China, 9-11 August.

Zhang, Aiwen Zhang; Min Zhou; Zhiping Cai; Huiying Xu, 2009. Design of a new-style and high-stability LD drive power supply, 3rd International Conference on Anti-counterfeiting, Security, and Identification in Communication (2009 ASID), pp. 238-41.

Zhou, Min; Aiwen Zhang; Zhiping Cai; Huiying Xu, 2009. Design of a highly stable LD power supply and a highly reliable protection circuit, Proceedings of the SPIE - The International Society for Optical Engineering, v 7506, pp. 750-616.

Zhou, Guoqing and Jiazhi Yang, 2011. Power supply topology for LiDAR system onboard UAV platform, Proceedings of SPIE - The International Society for Optical Engineering, International Symposium on Lidar and Radar Mapping 2011: Technologies and Applications, vol. 8286.

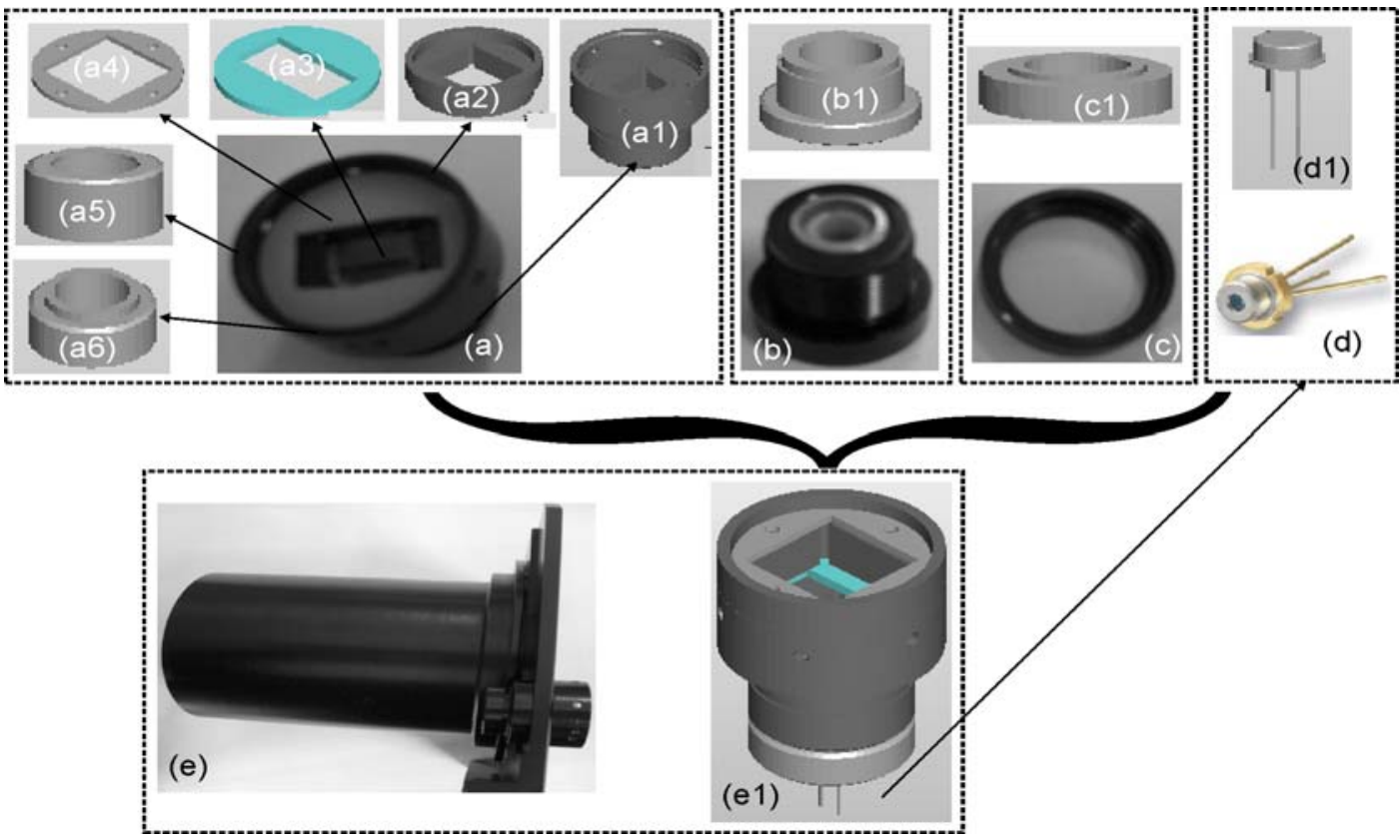

Figure 11. Hardware components of laser emitting system 